\title{
BMJ Open Does the impact of a plant-based diet during pregnancy on birth weight differ by ethnicity? A dietary pattern analysis from a prospective Canadian birth cohort alliance
}

\author{
Michael A Zulyniak, ${ }^{1,2}$ Russell J de Souza, ${ }^{3}$ Mateen Shaikh, ${ }^{3}$ Dipika Desai, ${ }^{4}$ \\ Diana L Lefebvre, ${ }^{1}$ Milan Gupta,,${ }^{1,5}$ Julie Wilson, ${ }^{6}$ Gita Wahi, ${ }^{3,7}$ Padmaja Subbarao, ${ }^{8}$ \\ Allan B Becker, ${ }^{9}$ Piush Mandhane, ${ }^{10}$ Stuart E Turvey, ${ }^{11}$ Joseph Beyene, ${ }^{3}$ \\ Stephanie Atkinson, ${ }^{7}$ Katherine M Morrison, ${ }^{7}$ Sarah McDonald, ${ }^{3}$ Koon K Teo, ${ }^{1,4}$ \\ Malcolm R Sears, ${ }^{1}$ Sonia S Anand, ${ }^{1,3,4}$ for the NutriGen Alliance investigators
}

To cite: Zulyniak MA, de Souza RJ, Shaikh M, et al. Does the impact of a plant-based diet during pregnancy on birth weight differ by ethnicity? A dietary pattern analysis from a prospective Canadian birth cohort alliance. BMJ Open 2017;7:e017753. doi:10.1136/ bmjopen-2017-017753

- Prepublication history and additional material for this paper are available online. To view these files, please visit the journal online (http://dx.doi. org/10.1136/bmjopen-2017017753).

Received 19 May 2017 Revised 29 September 2017 Accepted 2 October 2017

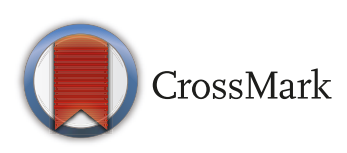

For numbered affiliations see end of article.

Correspondence to Professor Sonia S Anand; anands@mcmaster.ca

\section{ABSTRACT}

Objective Birth weight is an indicator of newborn health and a strong predictor of health outcomes in later life. Significant variation in diet during pregnancy between ethnic groups in high-income countries provides an ideal opportunity to investigate the influence of maternal diet on birth weight.

Setting Four multiethnic birth cohorts based in Canada (the NutriGen Alliance).

Participants 3997 full-term mother-infant pairs of diverse ethnic groups who had principal component analysis-derived diet pattern scores_-plant-based, Western and health-conscious — and birth weight data.

Results No associations were identified between the Western and health-conscious diet patterns and birth weight; however, the plant-based dietary pattern was inversely associated with birth weight $(\beta=-67.6 \mathrm{~g}$ per 1 -unit increase; $P<0.001$ ), and an interaction with non-white ethnicity and birth weight was observed. Ethnically stratified analyses demonstrated that among white Europeans, maternal consumption of a plant-based diet associated with lower birth weight ( $\beta=-65.9 \mathrm{~g}$ per 1 -unit increase; $\mathrm{P}<0.001$ ), increased risk of small-for-gestational age (SGA; $\mathrm{OR}=1.46 ; 95 \% \mathrm{Cl} 1.08$ to $1.54 ; \mathrm{P}=0.005)$ and reduced risk of large-for-gestational age (LGA; OR=0.71; $95 \% \mathrm{Cl} 0.53$ to $0.95 ; P=0.02)$. Among South Asians, maternal consumption of a plant-based diet associated with a higher birth weight ( $\beta=+40.5 \mathrm{~g}$ per 1-unit increase; $P=0.01$ ), partially explained by cooked vegetable consumption.

Conclusions Maternal consumption of a plant-based diet during pregnancy is associated with birth weight. Among white Europeans, a plant-based diet is associated with lower birth weight, reduced odds of an infant born LGA and increased odds of SGA, whereas among South Asians living in Canada, a plant-based diet is associated with increased birth weight.

\section{INTRODUCTION}

Birth weight is an indicator of infant health and a strong predictor of future health

\section{Strengths and limitations of this study}

- Prospective data collection, large sample size, adequate power to allow for ethnic comparisons and detailed measurement of diet using ethnic-specific Food Frequency Questionnaire are the strengths of the study.

- Limitations include self-reported retrospective food intake, the inability to adjust for differences in cooking methods and residual confounding, which is a potential bias in all cohort studies.

- To minimise potential bias, a sensitivity analysis was conducted, which excluded mothers who may have deliberately altered their diet due to a medical condition.

outcomes. ${ }^{1}$ Infants born small (birth weight $<10$ th percentile) or large (birth weight $\geq 90$ th percentile) for sex and gestational age are at increased risk of future health complications, including asthma, ${ }^{2}$ obesity ${ }^{3}$ and cardiovascular disease. ${ }^{4}$ High-income countries generally have similar proportions of babies who weigh $<2500 \mathrm{~g}$ at birth, but there is greater variation (up to $10 \%$ ) in the proportion of infants born $>4000 \mathrm{~g} .{ }^{5-7}$ However, such population figures often mask important differences in the distribution of birth weight between ethnic groups. In the USA, white Europeans have more high birth weight $(9.6 \%)$ than low birth weight infants $(7.0 \%)$; African-Americans have more low birth weight $(13.1 \%)$ than high birth weight infants $(4.3 \%)$; and Hispanics have an equal proportion $(7.1 \%$ high birth weight and $7.1 \%$ low birth weight infants). ${ }^{5}$ Furthermore, we recently reported that newborns in Canada 
of South Asian ancestry (those who originate from the Indian subcontinent) are of lower birth weight, and that Aboriginal newborns are of higher birth weight compared with full-term newborns of white European ancestry in Canada. ${ }^{8}$ This provides additional evidence that among full-term newborns, ethnic differences in birth weight distribution exist. Numerous factors influence birth weight, including gestational weight gain, maternal prepregnancy weight, maternal height, gestational diabetes and fetal smoke exposure. ${ }^{9}{ }^{10}$ It has been postulated that maternal dietary intake also influences birth weight. ${ }^{11}{ }^{12}$ The investigation of specific food items and nutrients has advanced our understanding of specific nutrient deficiency syndromes (eg, neural tube defects) and facilitated the identification of particularly harmful food components (eg, trans fats). However, single-nutrient studies may be misleading because they fail to capture the complex interplay between foods and nutrients consumed as meals over long periods of time. To overcome this, the empirical derivation of dietary patterns ${ }^{13}$ has been proposed as a method to characterise diet that more accurately reflects how we consume foods and nutrients, and these patterns can be assessed for their associations with health and disease.

In this study, we investigated the association between maternal diet and birth weight in a multiethnic cohort using the dietary pattern analysis approach.

\section{METHODS}

\section{Study population}

The NutriGen Alliance ${ }^{13}$ is a consortium of four birth cohorts in Canada investigating the contribution of nutritional, genetic and epigenetic factors to the health of pregnant women and their children-(1) Canadian Healthy Infant Longitudinal Development ${ }^{14}$ study (CHILD); (2) Family Atherosclerosis Monitoring In earLY life (FAMILY) study $^{15}$; (3) SouTh Asian birth cohoRT (START) ${ }^{16}$; and (4) Aboriginal Birth Cohort (ABC).${ }^{17}$ Of these, ABC is still recruiting. To date (October 2016), 5018 pregnant women have provided comprehensive clinical and dietary data and $4556(\approx 90.8 \%)$ have provided birth data. From this group, 559 women were excluded from the present analysis because they (1) reported an implausible energy intake ( $<500$ or $\geq 6500 \mathrm{kcal} /$ day), (2) were non-singleton pregnancies, (3) delivered preterm ( $<36$ weeks) and/or (4) reported not knowing their ethnic origin. Preterm infants were excluded to avoid confounding by pregnancy or neonatal complications (eg, trauma, stress, infections or placenta previa). The remaining 3997 fullterm infant-mothers represented multiple maternal self-reported ethnicities-white European $(n=2367)$, South Asian $(\mathrm{n}=884)$, East/South-East Asian $(\mathrm{n}=335)$, Aboriginal $(n=190)$, African $(n=60)$ and women who reported another ethnicity ( $\mathrm{n}=141$, eg, Egyptian, Haitian and others) (online supplementary efigure 1). Informed written consent was obtained from each participant within each study.

\section{Dietary assessment}

Dietary information during pregnancy was collected in each cohort 24-28 weeks' gestation using a validated semiquantitative Food Frequency Questionnaire (FFQ). The CHILD cohort used a version of the Fred Hutchinson Cancer Center tool. ${ }^{18}$ The FAMILY, START and ABC cohorts used ethnic-specific FFQs developed for the Study of Health Assessment and Risk in Ethnic groups. ${ }^{13} 1617$

\section{Diet pattern analysis}

Detailed methods of FFQ harmonisation and the statistical derivation of dietary patterns within the NutriGen cohort have been previously described. ${ }^{13}$ We performed principal component analysis (PCA) to identify three orthogonal dietary patterns-'plant-based', 'western' and 'health-conscious' (online supplementary etable 1). This approach retains the information of the quantitative data from the original FFQ but shifts the focus from individual food components to food combinations. An empirically derived measure of adherence (ie, loading scores) for each person to each of the individual dietary patterns is calculated in the PCA. The PCA loading scores for each individual were adjusted to the mean total population caloric intake (2500 $\mathrm{kcal} /$ day) using the residual method. ${ }^{19}$

\section{Clinical parameters}

The primary outcome of this analysis was newborn birth weight. Birth weight was collected from the birth chart or measured in duplicate by trained staff perinatally (median $<24$ hours; $95 \% \leq 48$ hours). Due to the ethnic diversity of the study population, sex-specific and ethnic-specific birth weight cut points were used to define infants born small (SGA $<10$ th percentile) or large (LGA $\geq 90$ th percentile) for gestational age, which demonstrate greater accuracy to identify risk in multiethnic cohorts. ${ }^{20}$ Gestational age was determined by ultrasound at the initial visit. Data on all clinical parameters are provided in table 1 .

\section{Statistical analysis}

The distribution of exposures and covariates was summarised using JMP (V.9.0.1) as means (SD) for continuous variables, or counts (\%) for categorical variables; between groups, differences were assessed using analysis of variance (continuous variables) or $\chi^{2}$ test (categorical variables).

Prepregnancy weights from the CHILD cohort were available for $\approx 73 \%$ of women. To impute missing weights, we constructed a multivariable model with white Europeans recruited in CHILD and FAMILY. The correlation between the value predicted by this equation and observed prepregnancy weight was 0.42 .

Due to the numerous biological, sociological and environmental variables that associate with birth weight, we aimed to identify a parsimonious set of covariates. All variables listed in table 1 significant by association with birth weight as the response variable in a simple linear regression $(\alpha \leq 0.10)$ were entered into a forward stepwise selection procedure. To account for perinatal reductions in weight, ${ }^{21}$ timing of birth weight measurement was 


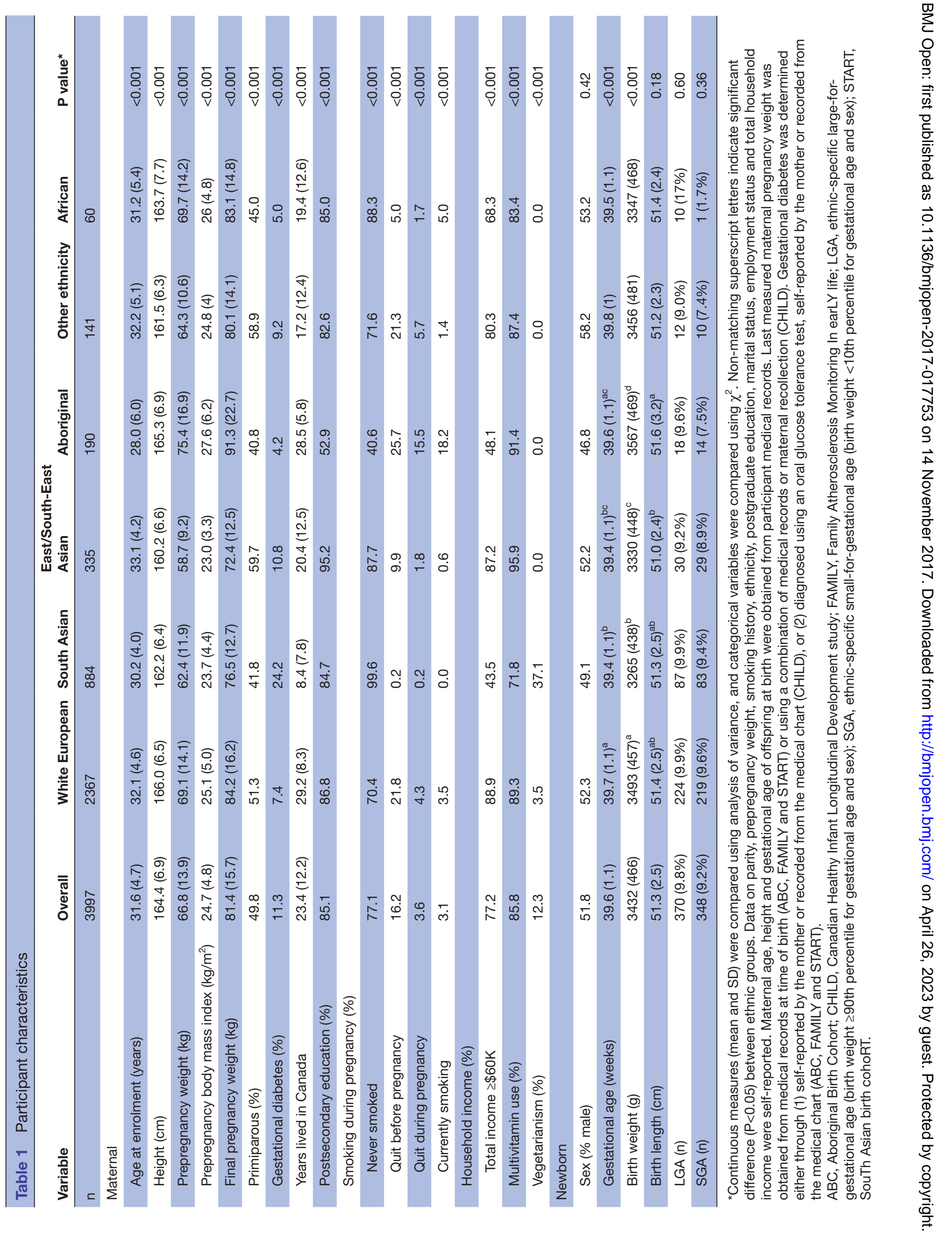


assessed as a covariate. Covariates significant at $\alpha<0.05$ in the stepwise multivariable model were retained as covariates in the final model.

Multivariable linear regression was used to assess the association between maternal dietary patterns and covariates on infant birth weight. The main effects, ethnicity and each of the dietary patterns were included in the initial model. Interaction terms between a dietary pattern and ethnicity were added if they were both significant at $\mathrm{P}<0.05$. To determine which foods contributed to the observed diet and birth weight association, food groups were added one at a time to the full dietary pattern-birth weight multivariable model. If the association between the dietary pattern and birth weight was rendered non-significant by the addition of the food group, we deemed it to be an important explanatory variable.

For ethnic-specific analyses, we had adequate power to investigate the association between plant-based diet and birth weight within the two largest ethnic groupswhite Europeans ( $>95 \%$ power) and South Asians $(\approx 80 \%$ power) - but $<40 \%$ power to do so in the other ethnic groups. Therefore, stratified analyses by ethnic group were limited to white Europeans and South Asians.

\section{RESULTS}

\section{Demographic and clinical parameters}

Maternal demographic and clinical parameters for each of the six ethnic groups are presented in table 1.

\section{Birth weight}

There were significant differences in birth weight by ethnic group $(\mathrm{P}<0.001)$. Aboriginal newborns had the highest birth weight, followed by white Europeans, other ethnicity, African origin, East/South-East Asians and South Asians (table 1).

\section{Effect of dietary patterns on birth weight}

In the fully adjusted main-effects multivariable model (table 2, model 1), non-white ethnicity and plant-based diet were significantly associated, while the Western and health-conscious diet patterns were not. After removal of non-significant dietary patterns, an interaction between the plant-based diet and non-white ethnicity was tested and found to be significant (table 2, model 2).

\section{Stratified analyses}

Multivariable models, stratified by ethnicity (white European and South Asian) and adjusted for the same covariates as the main model (except for smoking in South Asians, as its prevalence was $<0.5 \%$ ), were built, treating dietary pattern scores as either continuous (table 3) or dichotomous (fourth vs first quartile) variables (table 3). In white Europeans, an inverse association was observed between plant-based diet and birth weight. Among South Asians, a positive association was observed between the plant-based diet and birth weight (figure 1).

A sensitivity analysis, which excluded mothers who were diagnosed with gestational diabetes and/or hypertension, resulted in dietary associations that were largely unchanged,

Table 2 Final multivariable regression of the association and interaction between diet patterns in white and non-whites with birth weight $(g)$

\begin{tabular}{|c|c|c|c|c|}
\hline \multirow[b]{2}{*}{ Variables } & \multicolumn{2}{|l|}{ Model 1} & \multicolumn{2}{|l|}{ Model 2} \\
\hline & $\beta$ & $P$ value & $\beta$ & $P$ value \\
\hline Intercept & -4551.9 & $<0.001$ & -4560.8 & $<0.0001$ \\
\hline Maternal height (cm) & 8.4 & $<0.001$ & 8.5 & $<0.001$ \\
\hline Parity (number of children) & 67.6 & $<0.001$ & 66.0 & $<0.001$ \\
\hline Smoking status & -21.9 & 0.04 & -23.6 & 0.02 \\
\hline Infant sex (female=1) & -118.6 & $<0.001$ & -118.8 & $<0.001$ \\
\hline Non-white ethnicity & -33.5 & 0.04 & -28.1 & 0.08 \\
\hline Plant-based diet & -34.6 & $<0.001$ & -67.6 & $<0.001$ \\
\hline Western diet & -12.7 & 0.35 & ns & ns \\
\hline
\end{tabular}

Model 1 included all covariates, non-White ethnicity indicator, three diet patterns identified using PCA: plant-based, Western and healthconscious diet. Model 2 tests interactions between non-white ethnicity and significant diet patterns after removal of non-significant variables from model 1 ( $n=3997)$. Non-significant variables removed from the model are denoted as ' $n s$ '. Smoking status was ordinal and input as either $0=$ never smoked, $1=$ quit smoking prepregnancy, $2=$ quit smoking during pregnancy or $3=$ currently smoking. Parity was ordinal and reported as having $0,1,2,3,4,5$ or 6 (or more) children. Non-whites included individuals who self-reported as being of South Asian, East/South-East Asian or Aboriginal descent.

NA, not applicable; PCA, principal component analysis. 
Table 3 Multivariable regression of plant-based diet pattern (as a continuous measure) and birth weight stratified by ethnicity: white European and South Asians

\begin{tabular}{lllll}
\hline & White Europeans & & \multicolumn{2}{l}{ South Asians } \\
\cline { 2 - 3 } Variables & $\boldsymbol{\beta}$ & P value & $\boldsymbol{\beta}$ & P value \\
\hline Intercept & -4723.7 & $<0.001$ & -4248.1 & $<0.001$ \\
Prepregnancy weight (kg) & 5.1 & $<0.001$ & 5.3 & $<0.001$ \\
Maternal height (cm) & 9.3 & $<0.001$ & 6.0 & 0.01 \\
Parity (number of children) & 73.8 & $<0.001$ & 43.5 & 0.01 \\
Gestational age (weeks) & 159.3 & $<0.001$ & 155.6 & $\mathrm{~ns}$ \\
Smoking status & -37.6 & 0.002 & -98.3 & $<0.001$ \\
Infant sex (female=1) & -127.7 & $<0.001$ & 40.5 & $<0.001$ \\
Plant-based (continuous) & -65.9 & $<0.001$ & 0.01 \\
\hline
\end{tabular}

White European $r^{2}=0.246$, South Asian $r^{2}=0.178$. The same covariates were included for each ethnic-specific model, but non-significant covariates (eg, 'smoking' in South Asians) were removed in the final model (denoted as 'ns'). Smoking status was coded as follows: $0=n e v e r$ smoked, 1=quit smoking prepregnancy, 2=quit smoking during pregnancy or $3=$ currently smoking. Parity was reported as having $0,1,2,3,4,5$ or 6 (or more) children. White Europeans $(n=2367)$ and South Asians $(n=884)$.

with a negative association remaining in white Europeans $(\beta=-64.5 \mathrm{~g}$ per 1 -unit increase in score; $\mathrm{n}=2038 ; \mathrm{P}=0.002)$ and a positive association in South Asians $(\beta=38.3 \mathrm{~g}$ per 1-unit increase in score; $\mathrm{n}=728 ; \mathrm{P}=0.03)$.

In white Europeans, a 1-unit increase in plant-based diet score was associated with $\approx 50 \%$ increase in odds of SGA and $\mathrm{a} \approx 30 \%$ decrease in odds of LGA (table 4 ). In South Asians, a 1-unit increase in plant-based diet score presented a non-significant reduction in odds of SGA $(\mathrm{P}=0.428)$ and non-significant increase in odds of LGA $(\mathrm{P}=0.249) \quad($ table 4$)$.

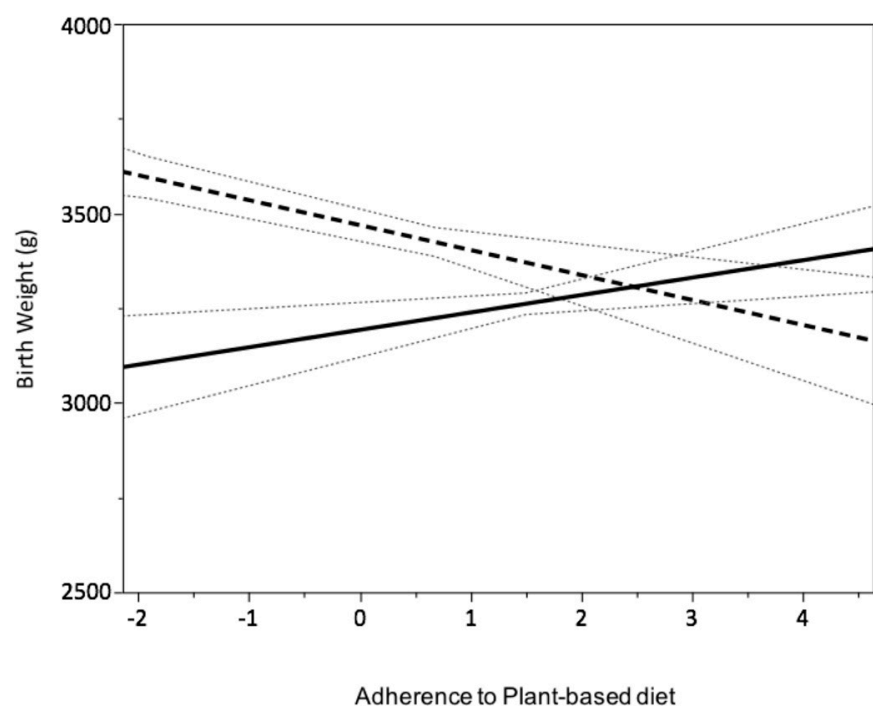

Figure 1 Multivariable regression of plant-based diet pattern and birth weight stratified by ethnicity. Association between maternal adherence to a plant-based diet, whereby a higher score reflects greater adherence to the dietary pattern, and birth weight in white Europeans (dashed line; $\mathrm{n}=2367$ ) and South Asians (solid line; $\mathrm{n=884}$ ) after accounting for covariates: prepregnancy weight, maternal height, maternal smoking status, number of children, and infant gestational age and sex. Dotted line $=95 \% \mathrm{Cl}$.

\section{Validity of the plant-based diet score}

The classification of individuals as plant-based consumers using the harmonised FFQ/PCA was validated against a classification using preharmonisation FFQ data. Using the raw number of servings obtained by each study-specific FFQ, the sum of servings for five plant-based foods groups common across all FFQs-that is, total dairy (an important protein source for lacto-vegtarians), vegetables, legumes, fruit, and breads and rice-was calculated. There was a high degree of agreement between preharmonised and postharmonised scores, with $>90 \%$ of the same plant-based consumers being classified in the fourth quartile using either metric $(\mathrm{P}<0.001)$.

\section{Diet comparison}

The food groups among individuals in the fourth quartile of the plant-based diet were compared between white Europeans and South Asians to understand the ethnic-specific structure of plant-based diets. For white Europeans, the plant-based diet was characterised by high intakes of fruit, nuts and seeds, convenience foods, sweet drinks, sweets and eggs, and moderate intakes of fish, poultry and red meat, while the South Asian plant-based diet was characterised by higher intakes of South Asian breads (eg, roti, naan), rice, legumes, raw vegetables and cooked vegetables (online supplementary etable 2). The use of multivitamins was comparable among high consumers of the plant-based diet in white Europeans (79\%) and South Asians (78\%), but differed in the low consumers of the plant-based diet, with white Europeans (94\%) more likely to take multivitamins than South Asians (66\%).

Among white Europeans, higher levels of postsecondary education, employment and marriage were observed in the fourth versus the first quartile of the plant-based diet, while South Asians in the fourth quartile were more likely to have recently immigrated to Canada, live with extended family in their household and were less likely to 
Table 4 Multivariable regression of plant-based diet pattern (as a categorical measure) and birth weight stratified by ethnicity: white European and South Asians

\begin{tabular}{|c|c|c|c|c|}
\hline \multirow[b]{2}{*}{ Variables } & \multicolumn{2}{|c|}{ White Europeans } & \multicolumn{2}{|c|}{ South Asians } \\
\hline & $\beta$ & $P$ value & $\beta$ & $P$ value \\
\hline Intercept & -5001.5 & $<0.001$ & -2482.3 & $<0.001$ \\
\hline Prepregnancy weight $(\mathrm{kg})$ & 4.1 & $<0.001$ & 6.8 & $<0.001$ \\
\hline Maternal height $(\mathrm{cm})$ & 9.4 & $<0.001$ & ns & ns \\
\hline Parity (number of children) & 77.1 & $<0.001$ & ns & ns \\
\hline Gestational age (weeks) & 168.6 & $<0.001$ & 135.6 & $<0.001$ \\
\hline Smoking status & ns & ns & ns & ns \\
\hline Infant sex (female $=1)$ & -130.4 & $<0.001$ & -122.8 & 0.002 \\
\hline Plant-based (fourth vs first quartile) & -81.2 & 0.002 & 70.5 & 0.07 \\
\hline
\end{tabular}

White European $r^{2}=0.246$, South Asian $r^{2}=0.155$. The same covariates were included for each ethnic-specific model, but non-significant covariates (eg, 'smoking' in South Asians) were removed in the final model (denoted as ' $n s$ '). Smoking status was coded as follows: $0=$ never smoked, 1=quitsmoking prepregnancy, 2=quit smoking during pregnancy or $3=$ currentlysmoking. Parity was reported as having $0,1,2,3,4,5$ or 6 (or more) children. White Europeans ( $n=2367)$ and South Asians $(n=884)$.

have postsecondary education compared with quartile 1 (online supplementary etable 2 ).

\section{Food groups}

Post-hoc analyses were conducted separately in white Europeans and South Asians, and identified 15 mutually exclusive food groups (online supplementary etable 2) that showed a high degree of variance between the individuals in the first and fourth quartiles of the plant-based diet. Only the addition of 'cooked vegetables' to the model reduced the magnitude of the plant-based dietary pattern association in South Asians $(\beta=37.9 \mathrm{~g} ; \mathrm{P}=0.041)$ (online supplementary etable 3 ). No other foods influenced the association of plant-based diet and birth weight in either ethnic group. Multivitamin use had no influence on the association.

\section{Effect of total energy intake on birth weight}

To investigate the effect of maternal energy intake on birth weight, we evaluated the association between total energy intake and birth weight in our population of white Europeans and South Asians with and without an interaction term for non-white ethnicity. Without ethnicity or its interaction term, we note there is a significant association between total maternal energy intake and infant birth weight, whereby a 100-calorie increase in total energy associated with $4 \mathrm{~g}(2-7 \mathrm{~g})$ increase in birth weight $(\mathrm{P}<0.01)$. When ethnicity and its interaction term (energy intake $\times$ non-white ethnicity) were included in the model, total energy intake and the interaction term were no longer significant $(\mathrm{P}>0.05)$, whereas ethnicity remained so $(\mathrm{P}<0.01)$, indicating that a South Asian newborn was estimated to weigh $231 \mathrm{~g}$ less $(-266$ to $-197 \mathrm{~g})$ than a white European newborn.

\section{DISCUSSION}

Among women living in a high-income country, ethnicity was a predictor of birth weight, independent of caloric intake, in white Europeans and South Asians. Foods and diet differ substantially between ethnic groups and may contribute to the association. A plant-based diet in pregnancy is associated with newborn birth weight, the effects of which are moderated by ethnicity. In white European women, consumption of a plant-based diet is associated with lower birth weight, an increased risk of an SGA infant and a reduced risk of an LGA infant, while in South Asian women the plant-based diet is associated with higher birth weight but had no significant effect on the risk of having an SGA or LGA infant.

Our findings are consistent with a recent systematic review that failed to find a consistent association of vegetarian diets on birth weight; five studies reported vegetarian mothers had lower birth weight babies, and two studies reported higher birth weights. ${ }^{22}$ The interpretation of the studies was complicated by (1) the numerous ethnic groups investigated, (2) differences in the foods that characterised the vegetarian and non-vegetarian (ie, control) groups within each of the reported studies, and (3) variability in the socioeconomic status (SES) of individuals on a vegetarian diet. Similar, in our study, birth weight was not associated with self-reported vegetarianism, which may be partly explained by the high degree of variability in the foods that comprise a plant-based diet across ethnic groups. This suggests that 'vegetarianism' may be too crude a descriptor of diet to allow for discovery of true associations with birth weight, because the definition of this term is qualitatively different across ethnic groups.

Studies investigating the effect of a plant-based diet in ethnically homogeneous populations are sparse. No study in pregnant South Asian women living in a high-income country has reported on dietary patterns and birth weight. However, our findings in white Europeans are consistent with previous work indicating that smoking is associated with reduced birth weight, ${ }^{23}$ an increased risk of SGA ${ }^{24}$ and reduced risk of $\mathrm{LGA},{ }^{25}$ and that increased parity, maternal 
weight and height are associated with a reduced risk of $\mathrm{SGA}^{26}$ and increased risk of LGA. ${ }^{25}$ Concerning diet, a previous study in white Europeans $(\mathrm{n}=38)^{27}$ reported that infants born to mothers whose diets were classified as 'plantbased' were lighter (3310g; 95\% CI 3080 to $3350 \mathrm{~g}$ ) than infants born to 'omnivore' mothers (3480 g; 95\% CI 3350 to $3620 \mathrm{~g})$. The Danish National Birth Cohort $(\mathrm{n}=44612)$ reported that mothers adhering to a plant-based dietary pattern were at reduced risk of having an SGA infant $(\mathrm{OR}=0.74 ; 95 \% \mathrm{CI} 0.64$ to 0.86$)$ compared with mothers adhering to a Western diet pattern. ${ }^{28}$ However, this observation may be confounded by the high prevalence of smoking during pregnancy in the Western diet $(38 \%)$ versus plantbased diet group (14\%).

Our investigation to determine which of the individual food groups characteristic of the plant-based diet may be driving the association revealed that controlling for cooked vegetables reduced the magnitude and significance of the association between the plant-based diet and birth weight in South Asians but not among white Europeans. In South Asians, cooked vegetables were independently associated with birth weight (after removing plant-based dietary pattern from the model), whereas multivitamin use was not. This suggests that consumption of 'cooked vegetables' may be a driver of the observed association between the plant-based diet and increased birth weight in South Asians.

Prior cohort studies from India have shown that lower levels of serum vitamin $\mathrm{B}_{12}{ }^{29}$ and folate in pregnancy are associated with low birth weight. ${ }^{30}$ In a randomised controlled trial, prenatal supplementation with vitamins A, D, E, C and B, as well as iron, zinc, copper, selenium and iodine, reduced the risk of low birth weight (Risk Ratio $(\mathrm{RR})=0.88 ; 95 \% \mathrm{CI} 0.85$ to 0.91$).{ }^{31}$ In the slum-dwelling population of Mumbai (India), micronutrient-rich snacks (green leafy vegetables, fruit and milk) consumed before or during pregnancy increase infant birth weight $(+48 \mathrm{~g}$; 95\% CI 1 to $96 \mathrm{~g}$ ) compared with a low-micronutrient snack (potato and onion). ${ }^{32}$ However, the dietary context in Canada differs substantially from that of low-income countries. Since 1998 flour and cereals have been fortified with folate. ${ }^{33}$ This, coupled with relatively high rates of multivitamin supplementation in pregnancy, makes folate and vitamin $\mathrm{B}_{12}$ deficiency in pregnancy unlikely among Canadians. In addition, women who avoid meat products yet consume milk, yoghurt, cheese and eggs usually attain adequate vitamin $B_{12}$. Thus, even in the context of virtually no dietary folate and vitamin $\mathrm{B}_{12}$ deficiency, high consumption of cooked vegetables as part of a traditional Indian diet is associated with higher birth weight compared with a lower plant-based/higher animal protein diet.

We can only speculate about the mechanisms underlying the association with cooked vegetables as we did not collect detailed information on cooking methods. In our study, South Asian women reported consuming a higher percentage of energy from fat than did white Europeans. Differences in food preparation methods can significantly alter the chemical and nutritional composition of dishes. ${ }^{34}$ This was recently examined using spectrophotometry to determine the chemical composition of fresh and fried portions of ovo-vegetarian dishes. ${ }^{35}$ Fried ovo-vegetarian dishes prepared in oil were lower in protein, fibre and carbohydrate content but higher in caloric and lipid content per $100 \mathrm{~g}$ serving, compared with fresh dishes. ${ }^{35}$ This aligns with work in pregnant South Asian women residing in East London (UK), who had a higher proportion of energy from fat (40\% energy) compared with other ethnic groups (white European, African and African-Caribbean). ${ }^{36}$ Further, a prospective birth study of 797 rural Indian women, reported that, although protein and carbohydrate intake at 18 weeks of gestation was unrelated to birth measurements, fat intake was associated with newborn length and adiposity. ${ }^{37}$ This suggests that South Asian cooking methods may alter the macronutrient composition of food by increasing the proportion of total fat relative to other nutrients, and offers a possible explanation regarding the difference in the association between the plant-based diet and birth weight in South Asians and white Europeans.

Although markers of SES were not significantly associated with birth weight, understanding differences in sociodemographic factors between high and low consumers of the plant-based diet is informative to understand the context in which these diets are consumed. In the Avon Longitudinal Study of Parents and Children (ALSPAC) birth cohort $(n=11833)$, SES was not associated with birth weight but was associated with food choices, whereby women of high SES consumed more healthy foods and less processed foods than women of a lower SES. ${ }^{23}$ In the present study, white European women who adhered most strongly to the plantbased diet represented the highest socioeconomic group compared with less adherent white European women. Interestingly this group consumed multivitamin supplements less frequently, and it is possible they perceived their 'healthy diet consumption' negated the need for the recommended daily supplement in pregnancy. In contrast, South Asian women who adhered most strongly to the plant-based diet were more recent immigrants to Canada, had lower income and were living with more extended family members, indicating more traditional cultural practices compared with the South Asian women who were low adherers to the plant-based diet. More recent immigrants with a high adherence to a plant-based diet also had greater multivitamin use compared with South Asians who were longer settled in Canada. The relationship between immigration and diet is complex due to the numerous and varied factors that influence acculturation within each ethnic group and country. ${ }^{38-40}$ Thus, policy recommendations for dietary guidelines should consider the socioeconomic profile of the population. Although recent evidence suggests that plant-based diets may improve health and reduce risk of disease, ${ }^{41}{ }^{42}$ our observations suggest that the food composition of the plant-based diets matters. Emphasis should likely be placed on whole foods, minimally processed and non-refined items. In light of this, 
dietary counselling and antenatal practitioners should tailor dietary guidance to match the SES and ethnicity of the patient whenever possible.

Our study has several strengths, including prospective data collection, large sample size, adequate power to allow for comparisons between ethnic groups, and detailed measurement of diet using validated, ethnic-specific FFQs. There are some limitations to our analyses, including self-reported retrospective food intake, the inability to account for differences in cooking methods and residual confounding, which is a potential bias in all cohort studies. To minimise this potential bias, we conducted a sensitivity analysis and excluded mothers who may have altered their diet due to a medical condition, such as diagnosed gestational diabetes or hypertension in pregnancy. After these exclusions, our results remained consistent.

\section{CONCLUSION}

Maternal consumption of a plant-based diet during pregnancy is associated with infant birth weight. Among white Europeans a plant-based diet is associated with lower birth weight, reduced odds of LGA and increased odds of SGA babies, whereas among South Asians living in Canada, a plant-based diet is associated with higher birth weight. Future studies are necessary to replicate these results and to elucidate potential mechanisms that underlie these ethnic-specific associations.

\section{Author affiliations}

${ }^{1}$ Department of Medicine, McMaster University, Hamilton, Ontario, Canada

${ }^{2}$ School of Food Science and Nutrition, University of Leeds, Leeds, UK

${ }^{3}$ Department of Health Research Methods, Evidence, and Impact, McMaster University, Hamilton, Ontario, Canada

${ }^{4}$ Population Health Research Institute, Hamilton Health Sciences and McMaster University, Hamilton, Ontario, Canada

${ }^{5}$ Canadian Cardiovascular Research Network, Brampton, Ontario, Canada

${ }^{6}$ Six Nations Health Services, Ohsweken, Ontario, Canada

${ }^{7}$ Department of Paediatrics, McMaster University, Hamilton, Ontario, Canada

${ }^{8}$ Hospital for Sick Children and Department of Paediatrics, University of Toronto,

Toronto, Ontario, Canada

${ }^{9}$ Department of Immunology, Faculty of Medicine, University of Manitoba, Winnipeg, Manitoba, Canada

${ }^{10}$ Department of Pediatrics, Faculty of Medicine and Dentistry, University of Alberta, Edmonton, Alberta, Canada

${ }^{11}$ Department of Paediatrics, Faculty of Medicine, University of British Columbia, BC Children's Hospital, Vancouver, British Columbia, Canada

Contributors SSA, ABB, JB, RJdS, DD, DLL, JW, PS, PM, SET, SA, MG, SM, KMM, MRS, KKT and GW conceptualised the study and acquired the data. MAZ, RJdS and SSA designed the study. MAZ, RJdS, MRS and SSA conceptualised and designed statistical modelling, conducted and interpreted the analysis, and drafted the manuscript. MAZ, RJdS, JB, MRS and SSA checked and validated statistical and mathematical assumptions. MAZ, RJdS, MRS and SSA prepared the final manuscript. All authors critically reviewed and approved the final manuscript.

Funding This work was supported by funding by a Canadian Institute for Health Research (CIHR) Grant in Food \& Health Population Health Research grant [RFA\# 201301FH6; 2013-2018; SAA, ABB, JB, RJdS, JAD, MG, SDM, AM, DM, KMM, GP, MRS, PJ, MGS, KKT, GW] and a CIHR RCT Fellowship grant [MTP201410; MAZ]. START data was collected as part of a bilateral ICMR /CIHR funded program [INC109205] and HSF Canada Grant in Aid [NA7283]. CIHR provided funds for CHILD [AEC85761; ABB, PM, MRS, PS, SET) with additional funding from AllerGen NCE Inc. for Nutrigen [SSA] and the CHILD Study (MRS). CIHR and HSF Canada provided funds for FAMILY [KKT], and ABC [SSA] data collection. MAZ was the recipient of a Young Investigator Award from the South Asian Network Supporting Awareness and Research (SANSAR). SDM holds a Canada Research Chair Tier II in in Maternal and Child Obesity Prevention and Intervention. MRS holds the Astra Zeneca chair in Respiratory Epidemiology. SSA holds a Canada Research Chair Tier 1 in Ethnic Diversity and Cardiovascular Disease and a Heart and Stroke Foundation/Michael G De Groote Chair in Population Health Research.

Competing interests None declared.

Ethics approval McMaster University. Ethical approval was obtained independently for all studies from the Hamilton Integrated Research Ethics Board-CHILD (REB 07-2929), FAMILY (REB 02-060), START (REB 10-640) and ABC (REB 12-152). Additional approval for $\mathrm{ABC}$ was obtained from the Six Nations Band Council.

Provenance and peer review Not commissioned; externally peer reviewed.

Data sharing statement Data sharing is currently not available for most data due to ethnical restrictions.

Open Access This is an Open Access article distributed in accordance with the Creative Commons Attribution Non Commercial (CC BY-NC 4.0) license, which permits others to distribute, remix, adapt, build upon this work non-commercially, and license their derivative works on different terms, provided the original work is properly cited and the use is non-commercial. See: http://creativecommons.org/ licenses/by-nc/4.0/

(c) Article author(s) (or their employer(s) unless otherwise stated in the text of the article) 2017. All rights reserved. No commercial use is permitted unless otherwise expressly granted.

\section{REFERENCES}

1. Mikolajczyk RT, Zhang J, Betran AP, et al. A global reference for fetalweight and birthweight percentiles. Lancet 2011;377:1855-61.

2. Mebrahtu TF, Feltbower RG, Parslow RC. Effects of birth weight and growth on childhood wheezing disorders: findings from the Born in Bradford Cohort. BMJ Open 2015;5:e009553.

3. Nordman H, Voutilainen R, Laitinen T, et al. Growth and cardiovascular risk factors in prepubertal children born large or small for gestational age. Horm Res Paediatr 2016;85:11-17.

4. Kernell K, Sydsjö G, Bladh M, et al. Congenital heart disease in men - birth characteristics and reproduction: a national cohort study. BMC Pregnancy Childbirth 2014:14:187.

5. Martin JA, Hamilton BE, Osterman MJ, et al. Births: final data for 2013. National vital statistics reports: from the centers for disease control and prevention, national center for health statistics. National Vital Statistics System 2015;64:1.

6. National Health Services. Hospital Episode Statistics: National Health Service.

7. Statistics Canada. CANSIM. Table 102-4509. Catalogue 8400210-X.

8. Oliveira AP, Kalra S, Wahi G, et al. Maternal and newborn health profile in a first nations community in Canada. J Obstet Gynaecol Can 2013;35:905-13.

9. Küpers LK, Xu X, Jankipersadsing SA, et al. DNA methylation mediates the effect of maternal smoking during pregnancy on birthweight of the offspring. Int J Epidemiol 2015;44:1224-37.

10. Kim SY, Sharma AJ, Sappenfield W, et al. Association of maternal body mass index, excessive weight gain, and gestational diabetes mellitus with large-for-gestational-age births. Obstet Gynecol 2014;123:737-44.

11. Knudsen VK, Heitmann BL, Halldorsson $\mathrm{TI}$, et al. Maternal dietary glycaemic load during pregnancy and gestational weight gain, birth weight and postpartum weight retention: a study within the danish national birth cohort. Br J Nutr 2013;109:1471-8.

12. Dwarkanath $P$, Barzilay JR, Thomas $T$, et al. High folate and low vitamin B-12 intakes during pregnancy are associated with smallfor-gestational age infants in South Indian women: a prospective observational cohort study. Am J Clin Nutr 2013;98:1450-8.

13. de Souza RJ, Zulyniak MA, Desai D, et al. Harmonization of foodfrequency questionnaires and dietary pattern analysis in 4 ethnically diverse birth cohorts. J Nutr 2016;146:2343-50.

14. Subbarao P, Anand SS, Becker AB, et al. The Canadian Healthy Infant Longitudinal Development (CHILD) Study: examining developmental origins of allergy and asthma. Thorax 2015;70:998-1000.

15. Morrison KM, Atkinson SA, Yusuf S, et al. The Family Atherosclerosis Monitoring In earLY life (FAMILY) study: rationale, design, and baseline data of a study examining the early determinants of atherosclerosis. Am Heart J 2009;158:533-9. 
16. Anand SS, Vasudevan A, Gupta M, et al. Rationale and design of South Asian Birth Cohort (START): a Canada-India collaborative study. BMC Public Health 2013;13:79.

17. Wahi G, Wilson J, Miller R, et al. Aboriginal birth cohort (ABC): a prospective cohort study of early life determinants of adiposity and associated risk factors among Aboriginal people in Canada. BMC Public Health 2013;13:608.

18. Fred Hutchinson Cancer Research Center. Food Frequency Questionnaires (FFQ). sharedresources.fredhutch.org. http:// sharedresources.fredhutch.org/services/food-frequencyquestionnaires-ffq (accessed 24 Jun2016).

19. Willett WC, Stampfer M. Willett W, eds. Implications of total energy intake for epidemiologic analyses: Nutritional epidemiology, 1998.

20. Hanley GE, Janssen PA. Ethnicity-specific birthweight distributions improve identification of term newborns at risk for short-term morbidity. Am J Obstet Gynecol 2013;209:428.e1-428.e6

21. Wright CM, Parkinson KN. Postnatal weight loss in term infants: what is normal and do growth charts allow for it? Arch Dis Child Fetal Neonatal Ed 2004;89:F254-F257.

22. Piccoli GB, Clari R, Vigotti FN, et al. Vegan-vegetarian diets in pregnancy: danger or panacea? a systematic narrative review. BJOG 2015;122:623-33.

23. Rogers I, Emmett $P$, Baker D, et al. Financial difficulties, smoking habits, composition of the diet and birthweight in a population of pregnant women in the South West of England. ALSPAC Study Team. Avon longitudinal study of pregnancy and childhood. Eur $J$ Clin Nutr 1998;52:251-60.

24. McCowan LM, Dekker GA, Chan E, et al. Spontaneous preterm birth and small for gestational age infants in women who stop smoking early in pregnancy: prospective cohort study. BMJ 2009;338:b1081-1.

25. Surkan PJ, Hsieh CC, Johansson AL, et al. Reasons for increasing trends in large for gestational age births. Obstet Gynecol 2004;104:720-6.

26. Thompson JM, Clark PM, Robinson E, et al. Risk factors for smallfor-gestational-age babies: The Auckland birthweight collaborative study. J Paediatr Child Health 2001;37:369-75.

27. Sanders TA, Reddy S. The influence of a vegetarian diet on the fatty acid composition of human milk and the essential fatty acid status of the infant. J Pediatr 1992;120:S71-S77.

28. Knudsen VK, Orozova-Bekkevold IM, Mikkelsen TB, et al. Major dietary patterns in pregnancy and fetal growth. Eur J Clin Nutr 2008;62:463-70.

29. Gammon CS, von Hurst PR, Coad J, et al. Vegetarianism, vitamin B12 status, and insulin resistance in a group of predominantly overweight/obese South Asian women. Nutrition 2012;28:20-4.
30. Muthayya S, Kurpad AV, Duggan CP, et al. Low maternal vitamin B12 status is associated with intrauterine growth retardation in urban South Indians. Eur J Clin Nutr 2006;60:791-801.

31. West KP, Shamim AA, Mehra S, et al. Effect of maternal multiple micronutrient vs iron-folic acid supplementation on infant mortality and adverse birth outcomes in rural Bangladesh: the JiVitA-3 randomized trial. JAMA 2014;312:2649-58.

32. Potdar RD, Sahariah SA, Gandhi M, et al. Improving women's diet quality preconceptionally and during gestation: effects on birth weight and prevalence of low birth weight-a randomized controlled efficacy trial in India (Mumbai Maternal Nutrition Project). Am J Clin Nutr 2014;100:1257-68.

33. De Wals $\mathrm{P}$, Tairou $\mathrm{F}$, Van Allen Ml, et al. Reduction in neural-tube defects after folic acid fortification in Canada. N Engl J Med 2007;357:135-42.

34. Lesser IA, Gasevic D, Lear SA. The association between acculturation and dietary patterns of South Asian immigrants. PLoS One 2014:9:e88495.

35. Barakat $\mathrm{H}$. Effect of frying-cooking on nutritional and bioactive compounds of innovative ovo-vegetarian diets. Food Nutr Sci 2014;05:1577-90.

36. Rees GA, Doyle W, Srivastava A, et al. The nutrient intakes of mothers of low birth weight babies - a comparison of ethnic groups in East London, UK. Matern Child Nutr 2005;1:91-9.

37. Rao S, Yajnik CS, Kanade A, et al. Intake of micronutrientrich foods in rural Indian mothers is associated with the size of their babies at birth: Pune Maternal Nutrition Study. J Nutr 2001;131:1217-24.

38. Wandel M, Råberg M, Kumar B, et al. Changes in food habits after migration among South Asians settled in Oslo: the effect of demographic, socio-economic and integration factors. Appetite 2008;50:376-85.

39. McDonald JT, Kennedy S. Is migration to Canada associated with unhealthy weight gain? overweight and obesity among Canada's immigrants. Soc Sci Med 2005;61:2469-81.

40. Sanou D, O'Reilly E, Ngnie-Teta I, et al. Acculturation and nutritional health of immigrants in Canada: a scoping review. J Immigr Minor Health 2014;16:24-34.

41. Aune D, Giovannucci E, Boffetta P, et al. Fruit and vegetable intake and the risk of cardiovascular disease, total cancer and all-cause mortality-a systematic review and dose-response meta-analysis of prospective studies. Int J Epidemiol 2017;46:1029-56.

42. Aune D, Keum N, Giovannucci E, et al. Whole grain consumption and risk of cardiovascular disease, cancer, and all cause and cause specific mortality: systematic review and dose-response metaanalysis of prospective studies. BMJ 2016;353:i2716. 